Original article

\title{
Psychosocial Factors That Have an Influence on the Effects of Obesity Improvement Programs
}

\author{
Hideaki Hanaoka ${ }^{1}$, Hitoshi Okamura ${ }^{1}$, Mamiko Iwamoto², \\ Chiaki Yagura ${ }^{3}$, Isao Kihara ${ }^{4}$, Akiko Nogi ${ }^{5}$, \\ Hajime Shimizu ${ }^{1}$ and Kuninori Shiwaku ${ }^{2}$ \\ ${ }^{1}$ Department of Physical Therapy and Occupational Therapy Sciences, Graduate School of Health Sciences, Hiroshima \\ University, Hiroshima 734-8551, Japan \\ ${ }^{2}$ Department of Environmental \& Preventive Medicine, Shimane University School of Medicine, Shimane 693-8501, Japan \\ ${ }^{3}$ Department of Physical Therapy, School of Rehabilitation Sciences at Fukuoka, International University of Health and Welfare, \\ Fukuoka 831-8501, Japan \\ ${ }^{4}$ Department of Life Science, Shimane University School of Medicine, Shimane 693-8501, Japan \\ ${ }^{5}$ Department of Human Nutrition, Faculty of Nursing and Human Nutrition, Yamaguchi Prefectural University, Yamaguchi 753- \\ 8520, Japan
}

\begin{abstract}
Objective: The objective of the present study was to clarify the relationship between factors having an influence on obesity improvement programs and psychosocial factors from a more comprehensive point of view.

Methods: We studied a total of 43 subjects with a body mass index (BMI) of $25 \mathrm{~kg} / \mathrm{m}^{2}$ or higher who wished to take part in an obesity improvement program and agreed to participate in the study. We conducted an obesity improvement program based on behavior change theories for three months and evaluated physical composition, mental health, social support, stress-coping and the like before intervention and immediately after completion of the program.

Results: The average weight showed a significant decrease from $69.0 \pm 8.8 \mathrm{~kg}$ to $65.7 \pm 8.7 \mathrm{~kg}$ before and after intervention $(p<0.001)$, respectively. It was also shown that the presence or absence of chronic diseases, social support from a spouse and the decrease of avoidance stress coping were related to weight loss.

Conclusion: The findings suggest that it will be further necessary to continue working on the need to enhance awareness about stress with a view to preventing occurrence of rebound after the end of weight loss programs and acquisition coping techniques, apart from the cooperation of attending doctors, strengthening of social support from family and friends and managing stress for the duration of the program.
\end{abstract}

Correspondence to: Hideaki Hanaoka

Department of Physical Therapy and Occupational Therapy Sciences, Graduate School of Health Sciences, Hiroshima University, 2-3

Kasumi 1-chome, Minami-ku, Hiroshima 734-8551, Japan

E-mail:hhanaoka@hiroshima-u.ac.jp
Key words: overweight, psychosocial, weight loss

(J Rural Med 2010; 5(2): 175-183)

\section{Introduction}

The worldwide increase of obesity and obesity-related diseases $^{1)}$ is an important matter of concern not only for Europe and America ${ }^{2,3)}$, but also for Asia, including Japan ${ }^{4,5}$. The commonly used method for reducing weight consists of trying to improve the life-style of the individual mainly by way of diet and exercise over the course of 3 to 6 months with the participation of professionals ${ }^{6,7)}$. Although this support yields some results, the reality is that it is not uncommon for subjects to drop out of the program or who continue with the program, but without positive results. Because each subject yields different results even if the same approach is applied, studies have been conducted hitherto on the effects of obesity improvement programs, in other words, on the factors that may influence weight change and maintenance, principally focusing on psychosocial factors ${ }^{8,9)}$.

Having applied an obesity improvement program to overweight adult subjects to study the influence of psychosocial factors thereon, Aston et al. ${ }^{10)}$ concluded that the higher the General Health Questionnaire (GHQ) scores of the subjects were before intervention, that is, the worse their mental health conditions were, the less weight they lost. Chiriboga et al. ${ }^{11)}$ stated also that anxiety and depression had an influence on weight loss. Wing et al. ${ }^{12)}$ on the other hand suggest the importance of social support, stating that female subjects 
accompanied by their spouses achieved better weight loss results, while male subjects did so by taking part in the program alone. Furthermore, Sakane et al. ${ }^{13)}$ divided subjects who had participated in an obesity improvement program into a group that had received stress management and a group that had not and studied the subsequent effects. They reported that the group having had stress management yielded better results, including loss of weight, and indicated accordingly the importance of stress coping for the duration of intervention. Notwithstanding the above, Hainer et al. ${ }^{14)}$ stated that depression did not contribute to causing weight change following an obesity improvement program, whereas Gierzewski ${ }^{15)}$ reported that there was not any association between social support from a spouse and loss of weight. There is no consensus of opinion about the issue.

As seen from the above, while some psychosocial factors, such as mental health, depression, anxiety, social support from spouse, and stress coping during a program, have been pointed out as psychosocial factors having an influence on obesity improvement programs, there are studies that disagree with such result. It can be said therefore that further investigation is warranted. To work out a more effective obesity improvement program, it will be necessary to elucidate factors that influence intervention and reflect them on the program. The objective of the present study was to elucidate from a more comprehensive point of view the connection of factors that affect obesity improvement programs with psychosocial factors.

\section{Materials and Methods}

\section{Subjects}

The subjects were residents of Shimane Prefecture, Japan, who ware at least 30 years of age. To recruit participants, we advertised in public relations magazines to solicit participation in the obesity improvement program and obtained informed consent in writing from the individuals persons interested in signing up. Of the individuals who gave informed consent, excluding those who did not fill out the questionnaire, we chose 43 individuals who had a body mass index (BMI) of over $25 \mathrm{~kg} / \mathrm{m}^{2}$ in check before intervention and on whom we could conduct a follow-up survey as the subjects of the present study. The study period was 3 months from August to October 2007. The present study was conducted with the approval of the ethics committee of Shimane University School of Medicine.

\section{Intervention method}

The present obesity improvement program ${ }^{16-18)}$ was based on behavior change theories in health learning and consisted of the following components:
(1) Recognition by the participant of risk factor related to obesity

(2) Improvement target of physical composition or blood biochemical value for the program

(3) Establishment of specific goals for action to achieve the improvement target

(4) Acquisition of techniques for changing life-style

(5) Development of process evaluation capability for action goals

(6) Strengthening of motivation by way of supportive group therapy and fellow support

The present program set a goal of a $5 \%$ weight reduction after 3 months of intervention, taking into account such bad influences as weight rebound phenomenon after weight loss. Before implementing the program, we took note of the history of disorders and present illnesses, performed electrocardiography and confirmed with the participants the following conditions for participation:

(1) Participants who were under treatment or observation in medical institutions on account of obesity related diseases such as hypertension and hyperlipemia should get permission from their attending doctors.

(2) Participants who were currently taking medication should not change the dose or the frequency for the duration of the program.

Before implementing the program, we took physical measurements, and examined the blood biochemistry and lifestyles of the participants in order to prompt their recognition of risk factors related to obesity. On the basis of the results, each participant consulted doctors and dietitians to determine a personal improvement target for physical measurements_and blood values. Specific action goals were decided upon consultation with physiotherapists and dietitians. In relation to the above (4) to (6), learning sessions and group activities were held three times in total with a frequency of once a month. Doctors and occupational therapists took part in these sessions, in which the participants declared their own targets and learned methods to solve the problems facing them. In addition, the participants learned to look within themselves and alleviate the psychological stress found both in their own and social lives.

\section{Measures}

We performed assessment twice, before the intervention and immediately after the end of the program.

\section{Basic attributes}

We assessed from the survey form the age, sex, occupation (including part-time jobs, and self-owned businesses such as the farming), presence or absence of a spouse, presence or absence of chronic diseases (affected by any of hypertension, hyperlipidemia, diabetes, hyperuricemia, apo- 
plexy, cardiovascular disease, gastrointestinal illness, respiratory or neoplasm), presence or absence of pain in the knee and/or in the lower back and so forth.

\section{Anthropometric}

After a 12-hour fast, we measured the height and weight of each patient uniformly and obtained the BMI values.

\section{Lifestyle assessment}

The participant's daily diet and amount of physical activity were investigated before and after the program. Information on the participant's daily diet was obtained using an established self-administered quantitative food frequency questionnaire ${ }^{19}$. Trained nutritionists asked each participant about their weekly frequency of food intake for the recent month. The investigation sheet was designed for Japanese people by the Working Committee for Health Guidelines of the Japanese Ministry of Health and Welfare. It investigates the average intake and frequency of intake for 1 month in terms of 30 kinds of food.

The physical activity level was investigated using the questionnaire sheets on such things as time and strenuousness of work, movement and commuting, and the intensity of daily activity was calculated. We measured the number of steps taken by the subjects using a pedometer (OMRON, HJ113, Kyoto, Japan) for 1 week before the intervention and during the program. The number of steps per day before the program was calculated from the average value of one week before the program started. The number of steps per day during the program was calculated from the average value during the program period.

The resting energy expenditure (REE) was measured only once before the intervention. After a 15-min rest period, REE was measured in the sitting position with nondispersive infrared analysis (VMB-002N, VINE, Tokyo, Japan). Using this measurement meter, the oxygen concentration in the expired breath was measured for $3 \mathrm{~min}$, and the calculated energy equivalent of oxygen volume was found to be $4.825 \mathrm{kcal} / \mathrm{L}^{20,21)}$.

The total energy expenditure (TEE) before the intervention was equal to REE $\times 0.9 \times$ intensity of daily activity. According to the standards that follow, the TEE after the intervention was calculated by adding the activity-associated energy expenditure that changed during the period of program to TEE before the intervention. The adding activityassociated energy expenditure was equal to energy expenditure in addition movement shown according to gender and weight (rough estimate value of man and woman of 20-29 years old) $\times 0.9$ (compensating rate of basal metabolism decrease due to aging) $\times$ execution time. The energy expenditure by walking was calculated based on the calorie that corresponds in "hurried step", the energy expenditure other than walking was calculated based on the calorie that corre- sponded to the self-declared movement, and the execution time was calculated from the activity record ${ }^{22}$.

In addition, we assessed the participants drinking and smoking habits with the investigation form.

4. Psychosocial assessment

1) Presence or absence of stress (before intervention only)

Using the questionnaire, we asked the patients if they felt stress at the time and evaluated the presence or absence of stress before intervention.

2) Jichi Medical School Social Support (JMS-SSS)

This social support scale was developed by Tsutsumi et $a l .{ }^{23)}$ for evaluation of self-report measures for social support with 28 -item questionnaires. One reply concerning expectations from support sources, such as a spouse, family and friends, is sought out of four alternatives, and the total score is worked out according to support source. When support source is not found, the score for the item is zero. Namely, it is shown that the higher is the score obtained, the more support can be expected. The reliability and validity have been confirmed $^{23)}$.

3) Stress-coping test for health check-up examinees

This stress-coping test was developed by Hosaka et al. ${ }^{24)}$ for evaluation of self-report measures for stress coping with a 12-item questionnaires. One reply is sought out of four alternatives, and four kinds of stress coping, active behavioral, distraction, passive resignation and avoidance, can be assessed. The higher the score obtained is, the more significant the tendency is to follow the respective pattern of coping action. The reliability and validity have been confirmed ${ }^{25)}$.

4) General Health Questionnaire (GHQ)

This is a general health questionnaire developed by Goldberg et al. ${ }^{26)}$ for evaluation of a self-report measure for mental health degree that consists originally of 60 items. In addition, we used a GHQ scoring method for evaluation that awards 0,0 , 1and 1 point, although the evaluation is one out of four. In addition to the total score, four factors, such somatic symptoms, anxiety and insomnia, social dysfunction and depression, should be assessed. The higher the scored number of points is, the worse the mental health state of the subject is. The reliability and validity of the Japanese version consisting of 28 items have been confirmed ${ }^{27}$.

\section{Statistical analysis}

First, we determined the basic attributes of the subjects and compared their physical measurements before and after intervention using the paired $t$-test. Then, in order to study factors related to weight change in the subjects, we took the weight change (after the end of program - before intervention) as a dependent variable and classified factors that might have an influence, such as age, body weight and the BMI before intervention, and psychosocial evaluation (before 
Table1 Baseline characteristics of the subjects ( $N=43)$

\begin{tabular}{|c|c|c|c|c|c|}
\hline & Mean (s.d.) & Frequency $(\%)$ & & Mean (s.d.) & Frequency $(\%)$ \\
\hline Basic attributes & & & Anthropometric & & \\
\hline Age (years) & $57.8(7.5)$ & & Weight (kg) & $69.0(8.8)$ & \\
\hline Sex & & & BMI Mean $\left(\mathrm{kg} / \mathrm{m}^{2}\right)$ & $27.9(2.2)$ & \\
\hline Male & & $14(32.6)$ & BMI classification & & \\
\hline Female & & $29(67.4)$ & Overweight $(25 \leq \mathrm{BMI}<30)$ & & $38(88.4)$ \\
\hline Aetas & & & Obese $(\mathrm{BMI} \geq 30)$ & & $5(11.6)$ \\
\hline $40 \mathrm{~s}$ & & $7(16.3)$ & Lifestyle factors & & \\
\hline $50 \mathrm{~s}$ & & $14(32.6)$ & Energy intake (kcal/day) & $2116.6(474.5)$ & \\
\hline $60 \mathrm{~s}$ & & $22(51.2)$ & Total energy expenditure (kcal/day) & $2455.2(587.1)$ & \\
\hline Occupation & & & Smoking habit & & \\
\hline Presence & & $25(58.1)$ & Presence & & $6(14.0)$ \\
\hline Absence & & $18(41.9)$ & Absence & & $37(86.0)$ \\
\hline Spouse & & & Drinking habit & & \\
\hline Presence & & $35(81.4)$ & Presence & & $18(41.9)$ \\
\hline Absence & & $8(18.6)$ & Absence & & $25(58.1)$ \\
\hline Chronic diseases & & & Psychosocial factors & & \\
\hline Presence & & $33(76.7)$ & Stress & & \\
\hline Absence & & $10(23.3)$ & Presence & & $38(88.4)$ \\
\hline Chronic condition & & & Absence & & $5(11.6)$ \\
\hline Hypertension & & $20(46.5)$ & Social support & & \\
\hline Hyperlipidemia & & $20(46.5)$ & Spouse & $21.0(11.0)$ & \\
\hline Diabetes & & $8(18.6)$ & Family & $20.0(15.0)$ & \\
\hline Hyperuricemia & & $6(18.6)$ & Friends & $26.4(5.7)$ & \\
\hline Apoplexy & & $3(7.0)$ & Stress coping & & \\
\hline Cardiovascular disease & & $7(16.3)$ & Active behavioral & $80.0(22.2)$ & \\
\hline Gastrointestinal illness & & $3(7.0)$ & Distraction & $36.6(9.1)$ & \\
\hline Respiratory & & $2(4.7)$ & Passive resignation & $28.4(7.0)$ & \\
\hline Neoplasm & & $2(4.7)$ & Avoidance & $45.6(9.6)$ & \\
\hline Pain in knee & & & GHQ28 & & \\
\hline Presence & & $13(30.2)$ & Total score & $5.6(4.9)$ & \\
\hline Absence & & $30(69.8)$ & Somatic symptoms & $2.2(2.0)$ & \\
\hline Pain in the lower back & & & Anxiety and insomnia & $1.9(1.8)$ & \\
\hline Presence & & $14(32.6)$ & Social dysfunction & $1.0(1.4)$ & \\
\hline Absence & & $29(67.4)$ & Depression & $0.4(1.3)$ & \\
\hline
\end{tabular}

intervention and its change) for examination of the Pearson correlation coefficient, and all other items into the 2 group. We then conducted a univariate analysis using the $t$-test and multiple regression analysis (forced entry method) with the related factors as independent variables. The $p$ values were on both sides, with correlation of $p<0.05$ in all examinations. We conducted all the statistical analyses using SPSS13.0J (Tokyo, Japan).

\section{Results}

Characteristics of the subjects (Table 1)

The ages of the subjects ranged from 42 to 69 years of age, with the average age being $57.8 \pm 7.5$ years. Among the participants, $67.4 \%$ were female, and the proportion of participants $\geq 60$-year-old was $51.2 \%$, half the number of subjects. In regards to occupations, $58.1 \%$ of the subjects ware engaged in some form of work, including part-time jobs. Although $81.4 \%$ of the participants had a spouse, only one spouse took part in the program and was accompanied by the other spouse. The average value of BMI was $27.9 \pm$ $2.2 \mathrm{~kg} / \mathrm{m}^{2}$, the number of subjects having a BMI of 30 or more was less $(11.6 \%)$ and most of the subjects were classified as overweight $\left(25 \leq \mathrm{BMI}<30 \mathrm{~kg} / \mathrm{m}^{2}\right)$.

Finally, only 5 of the 43 subjects replied that they did not feel stressed, while many of the participants felt stress in their daily lives. The average score of the GHQ28 was $5.6 \pm$ 4.9 points. 


\section{Effects of the obesity improvement program}

Energy intake showed a significant reduction, about $554.4 \mathrm{kcal} /$ day $(p<0.001)$, and fell from $2116.6 \pm 474.5 \mathrm{kcal} /$ day before intervention to $1562.3 \pm 307.8 \mathrm{kcal} /$ day after intervention. Also, the total energy expenditure showed a significant increase, about $208.5 \mathrm{kcal} / \mathrm{day}(p<0.001)$, and increased from $2455.2 \pm 587.1 \mathrm{kcal} /$ day before intervention to $2663.6 \pm 645.9 \mathrm{kcal} / \mathrm{day}$ after intervention $(p<0.001)$. The mean body weight also showed a significant reduction, about $3.3 \mathrm{~kg}(p<0.001)$, and fell from $69.0 \pm 8.8 \mathrm{~kg}$ before intervention to $65.7 \pm 8.7 \mathrm{~kg}$ after intervention. Accordingly, BMI also showed a significant reduction and fell from 27.9 $\pm 2.2 \mathrm{~kg} / \mathrm{m}^{2}$ before intervention to $26.6 \pm 2.4 \mathrm{~kg} / \mathrm{m}^{2}$ after intervention $(p<0.001)$.

\section{Factors related to weight loss (Table 2, 3)}

We studied the relationship among the weight change (weight after the end of the program - weight before intervention), basic attributes, psychosocial evaluation score before intervention and change of each evaluation score. Using a univariate analysis, we found that the presence or absence of chronic diseases, social support scores (spouse) before intervention, change in avoidance stress coping and depression before intervention were significantly related to the change in body weight (Table 2).

When we examined the relationships among these six factors, a significant correlation was shown between energy intake and energy expenditure. To avoid multicollinearity, we conducted a multiple regression analysis taking five factors (energy expenditure was not included) as independent variables. As a result, we obtained the factors having an influence on weight change: presence or absence of chronic diseases, social support scores (spouse) before intervention and change in avoidance stress coping (Table 3).

\section{Discussion}

\section{Change in weight reduction on account of the present program}

We recognized a significant change in body weight and BMI thanks to the intervention of the obesity improvement program, which prompted improvement mainly in terms of lifestyle. Whereas the National Heart, Lung, and Blood Institute recommends setting a weight reduction goal of about $10 \%$ within half a year taking into account such bad influences as the rebound phenomenon after completion of a weight loss program ${ }^{28)}$, we set goal of $5 \%$ weight reduction after 3 months of intervention in the present study. The average reduction of the subjects in the present study of about 3.3 $\mathrm{kg}$ (about 5\%) from a mean body weight of $69.0 \mathrm{~kg}$ before intervention suggests that the program's reduction effect was adequate.

\section{Factors having an influence on weight loss}

Having studied factors that might have an influence on weight change in the 3 -month weight loss program, we were able to show that subjects who had no chronic disease, but high expectations for social support from their spouse before intervention and less avoidance stress coping, which is a negative stress-coping method, lost more weight.

Chronic disease: Subjects without chronic diseases were shown to have experienced more weight reduction. Cheng et al. ${ }^{29)}$ elucidated that patients with more chronic diseases score lower with respect to health-related QOL (SF-36), especially the physical component summary (PCS), while Hung et al. reported that the PCS scores of overweight men with chronic diseases were lower than those of normal weight men. For this reason, it is reasonable to suggest that the PCS scores of the subjects having chronic diseases under the present study were reduced; that is, restriction of activities in jobs and daily lives was taking place.

Hanaoka et $\mathrm{ll}^{30)}$ studied factors related to weight loss in a weight loss program and reported that physical functioning (PF) and role physical (RP), which indicate the degree of physical health in relation to the health-related QOL (SF36), before intervention had an association with weight reduction. Therefore, it is possible to interpret that those subjects who find difficulty in physical activity in daily life cannot lose weight adequately. As chronic diseases causes degradation of physical activity, it is also thought to be difficult in the present study to enhance the reduced activity of subjects on account of chronic diseases in a short-term weight loss program, which suggests that chronic diseases may become one of the inhibitory factors.

Social support from spouse before intervention: Social support from spouse before intervention had a significant association with weight loss. The social support scale we used in the present study did not examine direct support but instead was one that reveals extent of support to be expected. Accordingly, those subjects who expected to receive support from their spouse in case of need without getting any actual support were the ones who were able to lose more weight.

The possible reason behind this is that the subjects could face the weight loss program, which might change their lifestyle with the thought that they could count on their spouses whenever they encountered problems in daily life, and this kind of thinking possibly enabled them to change their actions, resulting in effectual weight loss. Kayman et al. ${ }^{31}$ described the importance of having someone from whom to receive social support with a view to keeping control over one's body weight. In the meantime, Rabkin ${ }^{32)}$ reported that subjects taking part in a 12 -week weight loss program who 
Table 2 Factors related to weight loss - univariate analysis $(\mathrm{N}=43)$

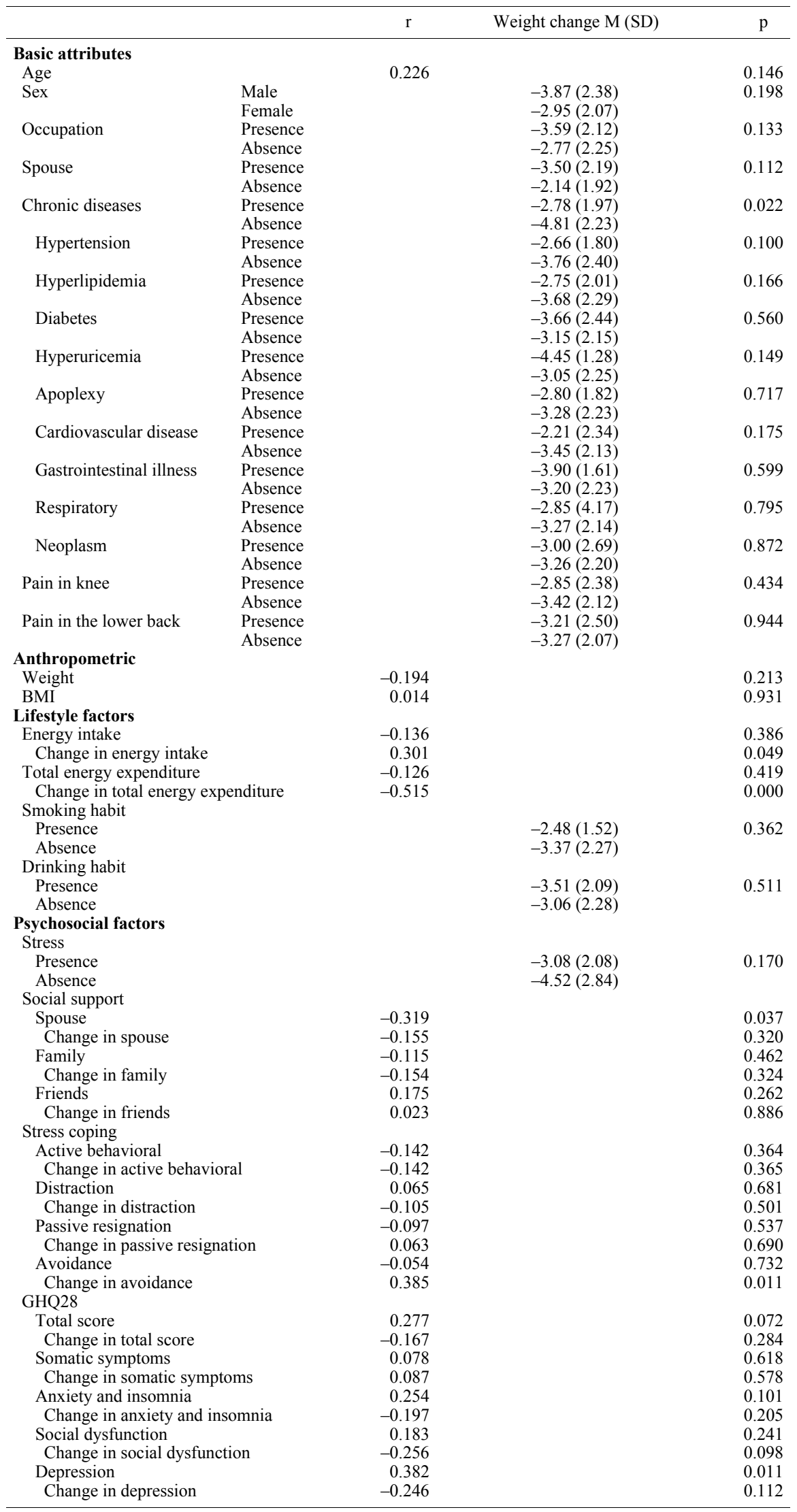


Table 3 Factors related to weight loss-multiple regression analysis $(\mathrm{N}=43)$

\begin{tabular}{lrrrr}
\hline & $\beta$ & $\mathrm{SE}$ & $\mathrm{t}$ & $p$ \\
\hline Chronic diseases & 0.296 & 0.729 & 2.081 & 0.044 \\
Change in energy intake & 0.012 & 0.001 & 0.081 & 0.936 \\
Social support scores (spouse) before intervention & -0.275 & 0.026 & -2.058 & 0.047 \\
Change in avoidance stress coping & 0.297 & 0.030 & 2.255 & 0.030 \\
Depression before intervention & 0.255 & 0.232 & 1.908 & 0.064 \\
\hline
\end{tabular}

$\mathrm{R}=0.649$. Adjusted $\mathrm{R}^{2}=0.343$.

replied before intervention that their spouses were collaborative in their efforts to reduce weight experienced more significant weight loss. The results of the present study support these findings.

In an earlier study on social support conducted on a gender basis, Wing et al. reported that the result was better for women when treated together with their spouses, while the result was better for men when they were treated alone. In the meantime, Elfhag et al. ${ }^{33)}$ concluded after a systematic review that intervention of the spouse is not necessarily positive and that there is a possibility that, in the long run, it may even impair the result. We are of the opinion therefore that there is still room left for study, such as on a gender basis.

Avoidance stress coping: We classified stress coping into active behavioral, distraction, passive resignation and avoidance and studied the relationship between each score before intervention and weight loss. While we could not find any significant connection, we observed that the change in avoidance coping after intervention was related significantly to weight loss. In other words, those subjects who stopped taking an avoidance coping style as a result of intervention managed to lose more weight regardless of their stress-coping attribute before intervention. Kayman et al ${ }^{31)}$ reported on the relationship between weight maintenance and stress coping and stated that there are many people with the tendency to gain weight who adopt an avoidance coping style, such as eating and drinking to cope with problems. In the case of the present study, it is thought that the decrease of such coping style reduced behavioral patterns such as eating and drinking to cope with stress, leading as a result to weight loss.

The issue in the future for the subjects who lost weight in the short-term program is to prevent them from regaining the weight after the end of the program. Sarlio-Lähteenkorva $e t$ $a l .{ }^{34)}$ reported that those who regained weight after losing it felt a great deal of stress in their daily lives compared with those who could maintain a normal weight. It is therefore important to learn to acquire a coping style, other than eating, that will not cause overeating to cope with stress.

\section{Limitations and future issues}

Our approach in this study has some limitations. The number of subjects was small, although they did have BMI values and weight-loss of participants of our intervention were lower than those of western countries, but BMI were similar to those reported in several population-based investigations in Japan, in line with our results. Further, it is known that short-term results do not necessarily continue in the long term, and our study was a short-term 3-month weight loss program. However, as our method included development of behavior, this may result in more enduring modification of behavior after the completion of the program, as compared with a program incorporating strict compliance with rules and a complicated prescription of lifestyle modification.

The present results suggest that subjects with chronic diseases should be provided with a certain degree of consideration, such as support for anxiety originating from changes in physical activity and eating habits, and cooperation from the attending doctors throughout the course of enrolment in a weight loss program. When we deal with subjects with a spouse on the other hand, we should consider the spouse as a source of social support; on the other hand, when dealing with subjects without a spouse, we should study the ways of strengthening social support from family and friends. Furthermore, it is necessary to dwell on stress management during the program, the awareness of stress for the purposes of avoiding the occurrence of rebound after the end of the program and the need for strengthening efforts to acquire coping techniques.

\section{Conclusion}

The present study ware also suggested that the existence or non-existence of chronic diseases, social support from a spouse and the decrease of avoidance stress coping are related to weight loss. In the future, it will be further necessary to continue working on the need to enhance the awareness about stress with a view to preventing occurrence of rebound after the end of weight loss programs and acqui- 
sition coping techniques, apart from the cooperation of attending doctors, strengthening of social support from family and friends and stress managing stress for the duration of the program.

\section{References}

1. Silventoinen $\mathrm{K}$, Sans $\mathrm{S}$, Tolonen $\mathrm{H}$, et al. Trends in obesity and energy supply in the WHO MONICA Project. Int J Obes 2004; 28: 710-718.

2. James PT. Obesity: the worldwide epidemic. Clin Dermatol 2004; 22: 276-280.

3. Ogden CL, Carroll MD, Curtin LR, et al. Prevalence of overweight and obesity in the United States, 1999-2004. JAMA 2006; 295: 1549-1555.

4. Huang IC, Frangakis C, Wu AW. The relationship of excess body weight and health-related quality of life: evidence from a population study in Taiwan. Int J Obes 2006; 30: 12501259.

5. Yoshiike N, Fujii H, Yoshita K. Epidemiology of obesity and overweight in Japanese children and Adults. Adiposcience 2004; 1: 48-55 (in Japanese, Abstract in English).

6. Fontaine KR, Barofsky I, Bartlett SJ, et al. Weight loss and health-related quality of life: results at 1-year follow-up. Eat Behav 2004; 5: 85-88.

7. Melanson KJ, Dell'Olio J, Carpenter MR, et al. Changes in multiple health outcomes at 12 and 24 weeks resulting from 12 weeks of exercise counseling with or without dietary counseling in obese adults. Nutrition 2004; 20: 849-856.

8. Teixeira PJ, Palmeira AL, Branco TL, et al. Who will lose weight? A reexamination of predictors of weight loss in women. Int J Behav Nutr Phys Act 2004; 1: 12.

9. Teixeira PJ, Going SB, Houtkooper LB, et al. Pretreatment predictors of attrition and successful weight management in women. Int J Obes 2004; 28: 1124-1133.

10. Anton SD, Martin CK, Redman L, et al. Psychosocial and behavioral pre-treatment predictors of weight loss outcomes. Eat Weight Disord 2008; 13: 30-37.

11. Chiriboga DE, Ma Y, Li W, et al. Gender differences in predictors of body weight and body weight change in healthy adults. Obesity 2008; 16: 137-145.

12. Wing RR, Jeffery RW. Benefits of recruiting participants with friends and increasing social support for weight loss and maintenance. J Consult Clin Psychol 1999; 67: 132-138.

13. Sakane N, Yosida T, Umekawa $\mathrm{T}$, et al. The significance of combined stress management therapy with diet and exercise in obese diabetic women with mental stress. J Jpn Diabetes Soc 1996; 39: 97-103(in Japanese, Abstract in English).

14. Hainer V, Hlavatá $\mathrm{K}$, Gojová $\mathrm{M}$, et al. Hormonal and psychobehavioral predictors of weight loss in response to a short-term weight reduction program in obese women. Physiol Res 2008; 57: S17-S27.

15. Gierszewski SA. The relationship of weight loss, locus of control, and social support. Nurs Res 1983; 32: 43-47.

16. Nogi A, Shiwaku K, Kitajima $\mathrm{K}$, et al. Weight loss and improbement of metabolic syndrome by intervention program based on health education. Journal of the Japanese Association of Rural Medicine (JJRM) 2004; 53: 649-659 (in Japanese, Abstract in English).

17. Shiwaku K, Nogi A, Anuurad E, et al. Difficulty in losing weight by behavioral intervention for women with Trp64Arg polymorphism of the beta3-adrenergic receptor gene. Int $\mathrm{J}$ Obes 2003; 27: 1028-1036.

18. Shiwaku K, Nogi A, Anuurad E, et al. Development and evaluation of intervention program for obesity based on health education and self-determination. Journal of the Japanese Association of Rural Medicine (JJRM) 2003; 52: 172-183 (in Japanese, Abstract in English).

19. Morimoto A, Takase Y, Hada K, et al. Evaluation of a "convenient method" for the estimation dietary food consumption. Jpn J Nutr 1977; 35: 235-245 (In Japanese, Abstract in English).

20. Li L, Ikenishi R, Iwamoto M, et al. Predictive values of anthropometric measurements for visceral obesity and metabolic syndrome in educational intervention. Journal of the Japanese Association of Rural Medicine (JJRM) 2008; 56: 852-862 (in Japanese, Abstract in English).

21. VINE Corp. The Instruction Manual of Nondispersive Infrared Analysis. VINE Corp, Tokyo, 2000.

22. Ainsworth BE, Haskell WL, Whitt MC, et al. Compendium of physical activities: an update of activity codes and MET intensities. Med Sci Sports Exerc 2000; 32 (9 Suppl): S498S504.

23. Tsutsumi A, Kayaba K, Ishikawa $\mathrm{S}$, et al. Jichi Medical School Social Support Scale (JMS-SSS) revision and tests for validity and reliability. Jpn J Public Health 2000; 47: 866-878 (in Japanese, Abstract in English).

24. Hosaka T, Takahashi T, Nakazato R, et al. Application of stress-coping test to health counseling center. Japanese Journal of Multiphasic Health Testing and Service 1998; 25: 270-274 (in Japanese).

25. Hosaka T, Hirai K, Sugiyama Y, et al. Study on the validity of the "stress-coping test" for health check-up examinees. Japanese Journal of Multiphasic Health Testing and Service 2001; 28: 11-15 (in Japanese, Abstract in English).

26. Goldberg DP, Hillier VF. A scaled version of the general health questionnaire. Psychol Med 1979; 9: 139-145.

27. Narita K. The factor structure of a general health questionnaire in a Japanese community sample —using a 28item version - . Jpn J Gerontol 1994; 16: 19-28 (in Japanese, Abstract in English).

28. The National Heart Lung and Blood Institute Obesity Education Initiative Expert Panel. Clinical Guidelines on the Identification, Evaluation, and Treatment of Overweight and Obesity in Adults. 1998; National Institute of Health Publication No. 98-4083: Bethesda.

29. Cheng L, Cumber S, Dumas C, et al. Health related quality of life in pregeriatric patients with chronic diseases at urban, public supported clinics. Health and Quality of Life Outcomes, 2003; 31: 63-70. 
30. Hanaoka H, Muraki T, Nogi A, et al. Effects of weight loss program on the health-related quality of life. General Rehabilitation 2005; 33, 951-957 (in Japanese).

31. Kayman S, Bruvold W, Stern JS. Maintenance and relapse after weight loss in women: behavioral aspects. Am J Clin Nutr 1990; 52: 800-807.

32. Rabkin SW. Psychosocial determinates of weight reduction in overweight individuals. J Obes Weight Regul 1983; 2: 97
106.

33. Elfhag K, Rössner S. Who succeeds in maintaining weight loss? A conceptual review of factors associated with weight loss maintenance and weight regain. Obes Rev 2005; 6: 6785 .

34. Sarlio-Lähteenkorva S, Rissanen A, Kaprio J. A descriptive study of weight loss maintenance: 6 and 15 year follow-up of initially overweight adults. Int J Obes 2000; 24: 116-125. 